\title{
The Effect of Choline Acetyltransferase Genotype on Donepezil Treatment Response in Patients with Alzheimer's Disease
}

\begin{abstract}
Kang Uk Lee, Jung Hie Lee ${ }^{1}$, Dong Young Lee, ${ }^{2,3}$, Jong Chul Youn ${ }^{4}$, Jeong Lan Kim ${ }^{5}$, Seok Woo Moon ${ }^{6}$, Bong-Jo Kim?', Seung-Ho Ryu', Moon Doo Kim ${ }^{9}$, Chang-Uk Lee ${ }^{10}$, Nam-Jin Lee ${ }^{11}$, Sung Man Chang ${ }^{12}$, Young Hoon $\mathrm{Kim}^{13}$, Do Hoon Kim ${ }^{14}$, Hae-Kook Lee ${ }^{15}$, Jong Inn Woo ${ }^{3}$, Ki Woong Kim ${ }^{3,16,17}$, Jin Hyeong Jhoo ${ }^{1}$

${ }^{1}$ Department of Psychiatry, Kangwon National University School of Medicine, Chuncheon, ${ }^{2}$ Department of Neuropsychiatry, Seoul National University Hospital, Seoul, ${ }^{3}$ Department of Psychiatry, Seoul National University College of Medicine, Seoul, ${ }^{4}$ Department of Neuropsychiatry, Kyunggi Provincial Hospital for the Elderly, Yongin, ${ }^{5}$ Department of Psychiatry, Chungnam National University Hospital, Daejeon, ${ }^{6}$ Department of Psychiatry, Konkuk University Chungju Hospital, Chungju, ${ }^{7}$ Department of Psychiatry, Gyeongsang National University Hospital, Jinju, ${ }^{8}$ Department of Neuropsychiatry, Konkuk University Hospital, Seoul, ${ }^{9}$ Department of Neuropsychiatry, Jeju National University Hospital, Jeju, ${ }^{10}$ Department of Psychiatry, Seoul St. Mary's Hospital, The Catholic University of Korea, Seoul, "Department of Psychiatry, Jeonju City Welfare Hospital for the Elderly, Jeonju, ${ }^{2}$ Department of Psychiatry, Kyungbook National University Hospital, Daegu, ${ }^{13}$ Department of Psychiatry, Haeundae Paik Hospital, Busan, ${ }^{14}$ Department of Psychiatry, Chuncheon Sacred Hospital, Chuncheon, ${ }^{15}$ Department of Psychiatry, Uijeongbu St. Mary's Hospital, Uijeongbu, ${ }^{16}$ Department of Brain and Cognitive Science, Seoul National University College of Natural Sciences, Seoul, ${ }^{17}$ Department of Neuropsychiatry, Seoul National University Bundang Hospital, Seongnam, Korea
\end{abstract}

\begin{abstract}
Objective: We examined the difference in responses to donepezil between carriers and non-carriers of the $\mathrm{A}$ allele at the +4 position of the choline acetyltransferase (ChAT) gene in Koreans.

Methods: Patients who met the criteria for probable Alzheimer's disease (AD) ( $n=199)$ were recruited. Among these, 145 completed the 12-week follow-up evaluation and 135 completed the 26-week scheduled course. Differences and changes in the Korean version of the mini-mental state examination (MMSE-KC) score, Korean version of the Consortium to Establish a Registry for Alzheimer's Disease Neuropsychological Assessment Battery (CERAD-K[N]) wordlist subtest score (WSS), CERAD-K(N) total score (TS), and the Korean version of geriatric depression scale (GDS-K) score between baseline and 12 weeks or 26 weeks were assessed by the Student's t-test.

Results: At 12 weeks, the changes in the MMSE-KC score, CERAD-K(N) WSS, and CERAD-K(N) TS from baseline were not significant between ChAT A allele carriers and non-carriers; however, at 26 weeks, these changes were significantly larger in ChAT A allele carriers than in non-carriers ( $p=0.02$ for MMSE-KC and $p=0.03$ for CERAD-K(N) WSS respectively). Conclusion: Our findings in this study suggested that presence of the A allele at the +4 position of ChAT might positively influence the treatment effect of donepezil in the early stages of $A D$ in Koreans.
\end{abstract}

KEY WORDS: Choline acetyltransferase; Single nucleotide polymorphism; Alzheimer disease; Donepezil.

\section{INTRODUCTION}

Cholinesterase inhibitors (ChEIs) were found to be effective for the treatment of mild to moderate Alzheimer's disease (AD). ${ }^{1)}$ Compared with placebo, they showed preventive effects on cognition, mood, and behavioral symptoms of patients with $\mathrm{AD}{ }^{1,2)}$ Unfortunately, their treatment effects are modest and not easy to detect in a

\footnotetext{
Received: January 7, 2015 / Revised: February 23, 2015

Accepted: February 24, 2015

Address for correspondence: Jin Hyeong Jhoo, MD, PhD Department of Psychiatry, Kangwon National University Hospital, 156 Baengnyeong-ro, Chuncheon 24289, Korea Tel: +82-33-254-9300, Fax: +82-33-244-2271 E-mail: jhoojh@kangwon.ac.kr
}

short-term duration. Furthermore, there might be substantial variability in treatment response and inter-patient variability in clinical response might be of special clinical importance for the vulnerable population taking ChEIs. Therapeutic response rates to ChEIs vary from $40 \%$ to $70 \%{ }^{3)}$

There is a strong need for development of biomarkers that can help identify individuals who would be more responsive to pharmacotherapy. As genetic factors could account for individual variability in drug deposition and pharmacodynamics, ${ }^{4)}$ many studies have investigated genetic variations as predictors of successful response to ChEIs. For instance, CYP2D6 polymorphism was associated with ChEIs treatment response, ${ }^{5,6)}$ and the presence

(a) This is an Open-Access article distributed under the terms of the Creative Commons Attribution Non-Commercial License (http://creativecommons.org/licenses/by-nc/4.0) which permits unrestricted non-commercial use, distribution, and reproduction in any medium, provided the original work is properly cited. 
of CYP2D6 gene variants conferring decreased or absent activity of the CYP2D6 enzyme was linked to better clinical response to donepezil. ${ }^{7,8)}$ The apolipoprotein $\mathrm{E}$ (APOE) epsilon4 allele was also reported to be associated with better clinical response to ChEIs in a couple of studies. ${ }^{9,10)}$ However, the impact of this allele was not consistently replicated. ${ }^{11)}$

A single nucleotide polymorphism (SNP) in choline acetyltransferase (ChAT) was also reported to be associated with response to ChEIs in patients with $\mathrm{AD}{ }^{12)}$ ChAT, an important enzyme in the synthesis of acetylcholine (Ach), is reduced in several brain regions of such patients, ${ }^{13)}$ and the reduction of ChAT correlated with the severity of dementia. ${ }^{14)}$ A SNP $(2384 \mathrm{G}>\mathrm{A})$ at the +4 position of the first common exon of ChAT (rs 3810950, Fig. 1) may influence its translation efficiency ${ }^{15)}$ and the association with AD risk has been reported in Koreans. ${ }^{16-18)}$ We selected this SNP among the ChAT SNPs based on these replicated findings. The effect of ChAT (2384G $>$ A) polymorphism on the clinical efficacy of ChEIs has not yet been fully investigated, although a previous report did not find a significant association between ChAT polymorphism and ChEIs treatment response. ${ }^{19)}$

This study aimed to investigate whether there is a significant difference in clinical responses to donepezil, as measured by cognitive function tests, between the A allele carriers and non-carriers at the +4 position of the ChAT gene in Koreans.

\section{METHODS}

\section{Participants}

This study was performed based on the 26 week, prospective and multi-center design. Fifteen hospitals participated between June 2006 and August 2008. We recruited patients who met the criteria for probable AD established by the National Institute of Neurological and Communicative Disorders and Stroke-Alzheimer's Disease and Related Disorders Association $^{20)}(\mathrm{n}=199)$, were 60-90 years of age, had Clinical Dementia Rating Scale (CDR) scores $^{21)}$ between 0.5 and 2, and Modified Hachinski Ischemic Scores $^{22)}$ below 4. Patients who met the following criteria were excluded: primary neurodegenerative disorders other than $\mathrm{AD}$, other severe neurological disorders of the brain, presence of severe mental illnesses including major depressive disorder or manic episodes, alcohol or substance dependence history, medical illness influencing cognitive functions, and the use of any ChEI during 3 months before donepezil treatment. Among 199 patients with $\mathrm{AD}, 145$ completed the 12-week follow-up evaluation and 135 completed the 26-week scheduled course.

Donepezil treatment was started with $2.5 \mathrm{mg}$ once a day and increased to $5 \mathrm{mg}$ a day after 1 week. The dose was increased to $10 \mathrm{mg}$ a day after 4 weeks if there was no adverse effect. Dosages of $5 \mathrm{mg}$ or $10 \mathrm{mg}$ a day were maintained for the rest of the study, based on tolerance and adverse responses to donepezil.

Collection and delivery of samples were managed by Seoul Medical Science Institute (Seoul, Korea) and ChAT genotyping for collected samples was done at Macrogen (Seoul, Korea).

The study protocol and informed consent forms were reviewed by the institutional review board and the patients or their guardians gave their informed consent to participate in the study.

\section{Assessment}

The Korean version of the Consortium to Establish a Registry for Alzheimer's Disease Neuropsychological Assessment Battery (CERAD-K[N]) including the Korean version of mini-mental state examination (MMSE-KC) ${ }^{23)}$ and the Korean version of Geriatric Depression Scale $(\text { GDS-K })^{24)}$ were administered at baseline as well as at 12 and 26 weeks for the evaluation of neurocognitive function and mood state. Based on CERAD-K(N), CERAD$\mathrm{K}(\mathrm{N})$ wordlist subset scores (WSS) that consisted of wordlist memory, wordlist recall, and wordlist recognition $^{25)}$ and CERAD-K(N) total scores (TS) that consisted of verbal fluency, Boston naming test, wordlist memory, construction praxis, wordlist recall, and wordlist recognition $^{26)}$ were calculated. CERAD-K(N) was performed by clinical psychologists or trained nurses in each hospital. CDR was also administered at baseline for evaluation of overall dementia severity. All clinical evaluations were performed blind to the results of the genotyping. Genotyping of APOE and ChAT was performed as described in our earlier study. ${ }^{27)}$ In brief, after genomic DNA was extracted from venous blood, upstream (AACCCTG GTGGATTTGGATT) and downstream (ATTTCCTTG GCACCCTGAG) primers were constructed to cover the first common coding exon of human ChAT gene including the position 4 based on the sequence of the human gene for ChAT (X56585). The PCR product was directly used in the sequencing reaction to determine the genotype. An automated sequencing reaction was performed using the Applied Biosystems ABI 3700 sequencer (Applied Biosystems, Foster City, CA, USA) in Macrogen. The 
genotype of the position +4 was determined by the electropherogram peak. APOE genotyping was performed according to the method previously described. ${ }^{28)}$

\section{Statistical Analysis}

Analysis was performed on 148 and 135 patients who completed a 12-week and 26-week follow-up evaluation respectively. The subjects were divided according to the presence or absence of the ChAT A allele at the +4 position of the first common exon of ChAT. Student's $t$-test was used to evaluate the difference in age, education level, MMSE-KC, CERAD-K(N) WSS, CERAD-K(N) TS, and the GDS-K scores between the ChATA allele carriers and non-carriers. Student's t-test was also used to evaluate the differences in the final dose of donepezil. The $\chi^{2}$ test was used to evaluate the baseline differences in sex and CDR scores.

Differences of changes in the MMSE-KC, CERAD$\mathrm{K}(\mathrm{N})$ WSS, CERAD-K(N) TS and GDS-K scores from baseline to 12 weeks or 26 weeks between the ChAT A allele carriers and non-carriers were assessed by Student's $t$-test with no covariates, because important covariates like age, sex, education, and APOE genotype were not different between two groups. Differences of changes in the above mentioned scores were also assessed by the Student's $t$-test between APOE $\varepsilon$ allele carriers and non-carriers. Statistical analyses were performed using IBM SPSS Statistics version 21.0 (IBM Co., Armonk, NY, USA). ChAT genotypes for 135 patients of 26 weeks were in Hardy-Weinberg equilibrium.

\section{RESULTS}

The 199 participants at the beginning of the study consisted of 64 men and 135 women. Their mean age was $74.6 \pm 6.6$ years and mean education level was $5.2 \pm 4.9$ years. The 148 participants evaluated at week 12 consisted of 46 men and 102 women. Their mean age was $74.6 \pm 6.5$ years and mean education level was $5.0 \pm 4.7$ years. The 135 participants evaluated at week 26 consisted of 44 men and 91 women. Their mean age was $74.2 \pm 6.5$ years and mean education level was $5.1 \pm 4.7$ years.

The demographic and clinical characteristics of the study populations at weeks 12 and 26 are described in Table 1. ChAT A allele carriers accounted for 47 of the 148 patients at week 12 and 41 of the 135 patients at week 26 . There was no significant difference in age, sex, education, and APOE e4 allele carrying status between ChAT A allele carriers and non-carriers at weeks 12 and 26. In addition, there was no significant difference in CDR, MMSE-KC, CERAD-K(N) WSS, CERAD-K(N) TS, and GDS-K scores at baseline between ChAT A allele carriers and non-carriers at weeks 12 and 26.

The changes of MMSE-KC, CERAD-K(N) WSS, and CERAD-K(N) TS at 12 weeks from baseline in ChAT A allele carriers were not significantly different compared with ChAT A allele non-carriers. The group difference scores in these changes between ChAT A allele carriers and non-carriers were $0.8,0.4$, and -0.1 respectively (Table 2 and Fig. 1).

The changes of MMSE-KC and CERAD-K(N) WSS at 26 weeks from baseline in ChAT A allele carriers were significantly different compared with ChAT A allele non-carriers ( $p=0.02$ and $p=0.03$ ). The change of CERAD-K(N) TS was not significantly different, but a meaningful difference was suggested $(p=0.09$ ). The group difference scores in the change of MMSE-KC, CERAD-K(N) WSS and CERAD-K(N) TS were 1.4, 2.4, and 2.7 respectively (Table 2 and Fig. 2). Power estimates for detecting the difference in the changes of MMSE-KC, CERAD-K(N) WSS and CERAD-K(N) TS according to the ChAT A allele carrying status at 26 weeks in this study were 0.56 , 0.65 and 0.41 respectively. The changes of GDS scores at 12 and 26 weeks from baseline were not significantly different between the two groups (Table 2).

There were no significant differences in the changes of

Table 1. Demographic and clinical characteristics of study subjects at baseline

\begin{tabular}{|c|c|c|c|c|}
\hline & \multicolumn{2}{|c|}{ Patients at week $12(n=148)$} & \multicolumn{2}{|c|}{ Patients at week $26 \quad(n=135)$} \\
\hline & $\begin{array}{l}\text { ChAT A allele } \\
\text { Carriers }(n=47)\end{array}$ & $\begin{array}{c}\text { ChAT A allele } \\
\text { Non-carriers }(n=101)\end{array}$ & $\begin{array}{l}\text { ChAT A allele } \\
\text { Carriers }(n=41)\end{array}$ & $\begin{array}{c}\text { ChAT A allele } \\
\text { Non-carriers }(n=94)\end{array}$ \\
\hline Age $(y r)$ & $74.6 \pm 6.2$ & $74.6 \pm 6.7$ & $74.1 \pm 6.2$ & $74.3 \pm 6.7$ \\
\hline Sex (male : female) & $12: 35$ & $34: 67$ & $11: 30$ & $33: 61$ \\
\hline Education (yr) & $4.7 \pm 4.1$ & $5.1 \pm 4.9$ & $4.7 \pm 4.1$ & $5.3 \pm 4.9$ \\
\hline ChAT (A/A : A/G) & $1: 46$ & & $1: 40$ & \\
\hline APOE $\varepsilon 4$ carriers & 17 & 39 & 13 & 37 \\
\hline CDR $0.5: 1: 2$ & $24: 20: 3$ & $49: 46: 6$ & $21: 17: 3$ & $45: 43: 6$ \\
\hline
\end{tabular}

Values are presented as mean \pm standard deviation or number only.

ChAT, choline acetyltransferase; APOE, apolipoprotein E; CDR, Clinical Dementia Rating Scale. 
Table 2. MMSE-KC, CERAD-K(N) WSS, CERAD-K(N) TS and GDS-K scores in study populations at baseline, 12 weeks and 26 weeks according to ChAT +4 A allele carrying status

\begin{tabular}{|c|c|c|c|c|c|c|c|c|}
\hline & \multicolumn{4}{|c|}{ Patients at 12 week $(n=148)$} & \multicolumn{4}{|c|}{ Patients at week $26 \quad(n=135)$} \\
\hline & Baseline & Week 12 & $\begin{array}{c}\Delta \text { (week } \\
\text { 12-baseline) }\end{array}$ & $\begin{array}{c}\text { Group } \\
\text { difference }\end{array}$ & Baseline & Week 26 & $\begin{array}{c}\Delta \text { (week } \\
\text { 26-baseline) }\end{array}$ & $\begin{array}{l}\text { Group } \\
\text { difference }\end{array}$ \\
\hline $\begin{array}{l}\text { MMSE-KC } \\
\text { ChAT A carriers } \\
\text { ChAT A non-carriers }\end{array}$ & $\begin{array}{l}17.2 \pm 4.4 \\
17.4 \pm 4.7\end{array}$ & $\begin{array}{l}18.1 \pm 5.0 \\
17.6 \pm 5.0\end{array}$ & $\begin{array}{l}1.0 \pm 3.0 \\
0.2 \pm 2.7\end{array}$ & 0.8 & $\begin{array}{l}17.4 \pm 4.5 \\
17.6 \pm 4.6\end{array}$ & $\begin{array}{l}18.7 \pm 4.5 \\
17.5 \pm 5.5\end{array}$ & $\begin{array}{r}1.3 \pm 2.9 \\
-0.1 \pm 3.5\end{array}$ & 1.4, $p=0.02$ \\
\hline $\begin{array}{l}\text { CERAD-K(N) WSS } \\
\text { ChAT A Carriers } \\
\text { ChAT A non-carriers }\end{array}$ & $\begin{array}{l}16.3 \pm 9.9 \\
14.6 \pm 7.4\end{array}$ & $\begin{array}{l}18.7 \pm 9.6 \\
16.5 \pm 8.1\end{array}$ & $\begin{array}{l}2.3 \pm 5.4 \\
1.9 \pm 5.2\end{array}$ & 0.4 & $\begin{array}{r}16.0 \pm 10.0 \\
14.8 \pm 7.5\end{array}$ & $\begin{array}{r}19.8 \pm 10.1 \\
16.1 \pm 8.6\end{array}$ & $\begin{array}{l}3.8 \pm 6.7 \\
1.4 \pm 5.4\end{array}$ & 2.4, $p=0.03$ \\
\hline $\begin{array}{l}\text { CERAD-K(N) TS } \\
\text { ChAT A Carriers } \\
\text { ChAT A non-carriers }\end{array}$ & $\begin{array}{l}39.0 \pm 16.0 \\
36.0 \pm 12.2\end{array}$ & $\begin{array}{l}41.7 \pm 15.6 \\
38.8 \pm 13.6\end{array}$ & $\begin{array}{l}2.7 \pm 8.1 \\
2.8 \pm 7.8\end{array}$ & -0.1 & $\begin{array}{l}39.2 \pm 16.7 \\
36.5 \pm 12.1\end{array}$ & $\begin{array}{l}44.2 \pm 16.0 \\
38.8 \pm 14.7\end{array}$ & $\begin{array}{l}5.1 \pm 8.9 \\
2.4 \pm 8.2\end{array}$ & 2.7, $p=0.09$ \\
\hline $\begin{array}{l}\text { GDS-K } \\
\text { ChAT A carriers } \\
\text { ChAT A non-carriers }\end{array}$ & $\begin{array}{l}13.4 \pm 7.4 \\
12.2 \pm 6.3\end{array}$ & $\begin{array}{l}12.0 \pm 7.0 \\
11.6 \pm 5.8\end{array}$ & $\begin{array}{l}-1.4 \pm 6.6 \\
-0.7 \pm 5.6\end{array}$ & -0.7 & $\begin{array}{l}12.5 \pm 6.8 \\
12.3 \pm 6.0\end{array}$ & $\begin{array}{l}11.7 \pm 6.8 \\
11.7 \pm 6.3\end{array}$ & $\begin{array}{l}-0.8 \pm 8.3 \\
-0.6 \pm 6.6\end{array}$ & -0.2 \\
\hline
\end{tabular}

MMSE-KC, Korean version of Mini-Mental State Examination; CERAD-K(N), Korean version of the Consortium to Establish a Registry for Alzheimer's Disease Neuropsychological Assessment Battery; WSS, wordlist subtest score; TS, total score; GDS-K, Korean version of Geriatric Depression Scale; ChAT, choline acetyltransferse.

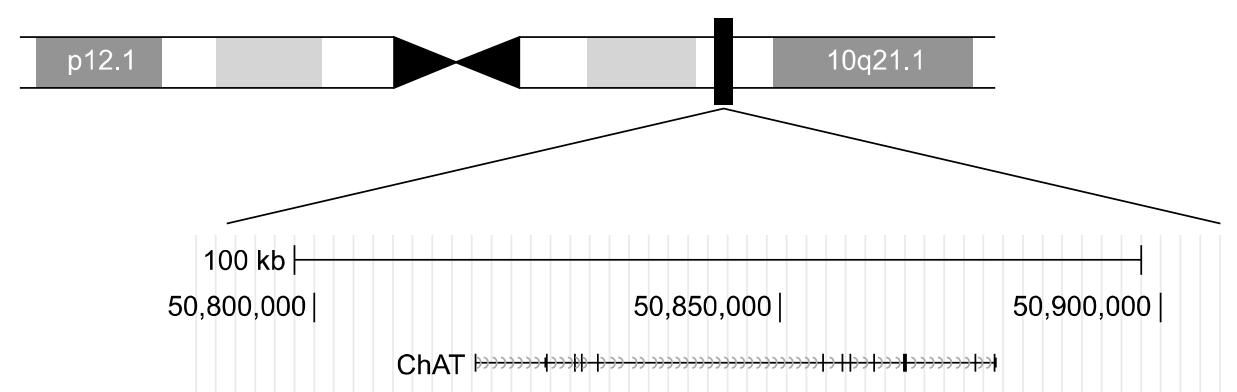

Fig. 1. The location and size of choline acetyltransferase gene in chromosome 10.

ChAT, choline acetyltransferase (source: genome.ucsc.edu).
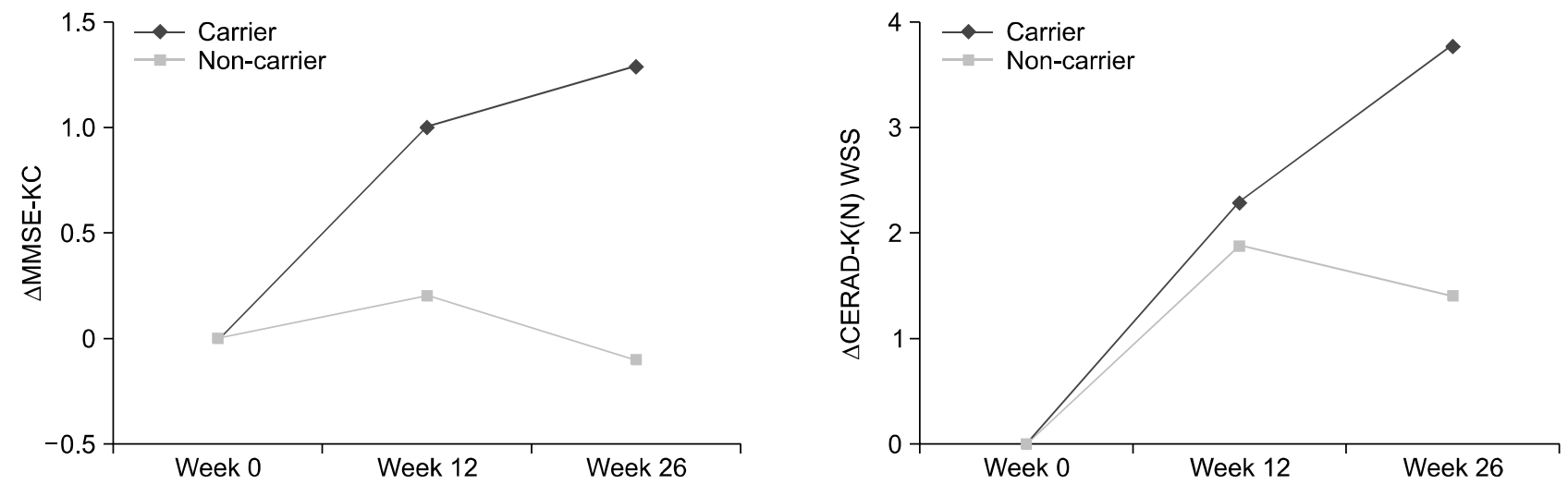

Fig. 2. Change of MMSE-KC and CERAD-K (N) wordlist subtest scores (WSS) from baseline to 12 and 26 weeks during the donepezil treatment according to the ChAT A allele carrying status.

MMSE-KC, Korean version of Mini-Mental State Examination; CERAD-K(N), Korean version of the Consortium to Establish a Registry for Alzheimer's Disease Neuropsychological Assessment Battery; WSS, wordlist subset scores.

MMSE-KC, CERAD-K(N) WSS, CERAD-K(N) TS, and GDS-K scores between APOE $\varepsilon 4$ allele carriers and non-carriers at 12 and 26 weeks from baseline (data was not shown). The mean donepezil dosage at the end of the study in ChAT A allele carriers and non-carriers was $9.4 \pm 1.6$ and $9.7 \pm 1.1$ respectively and there was no significant difference in dosage levels.

\section{DISCUSSION}

We found ChAT +4 A allele carriers had better treatment responses to donepezil than non-carriers among patients with $\mathrm{AD}$ at 26 weeks from baseline. This finding suggests that the ChAT genotype might be a potential biomarker for the prediction of successful ChEI response. 
There are reports of reduced cortical ChAT activity widespread brain regions of patients with $\mathrm{AD}^{13,29)}$ and this reduction correlated with $\mathrm{AD}$ severity. ${ }^{14)}$ In degenerative neural diseases like $\mathrm{AD}$ which involve a significant decrease in ChAT and Ach levels, different translation efficiency of ChAT gene variants might influence the Ach level and the effect of ChEI inhibitor. In AD, a SNP $(2384 \mathrm{G}>\mathrm{A})$ at the +4 position of the first common exon of ChAT might be a risk factor since A allele carriers had a significantly earlier age of onset and worse individual cognitive functioning. ${ }^{18,30)}$ In addition, the ChAT $+4 \mathrm{~A}$ allele may influence the translation efficiency of ChAT. ${ }^{15)}$ Kozak $^{15)}$ reported that the recognition of the initiator codon improves when $\mathrm{G}$ is substituted for $\mathrm{A}$ in +4 position. Even though it is not the rate-limiting enzyme, substitution of A allele instead of $\mathrm{G}$ might reduce the ChAT production and its role could be potentiated in specific states like $\mathrm{AD}$ and altered production due to such a polymorphism could be related to the treatment effect of donepezil.

Scacchi et al. ${ }^{19)}$ found no significant association between ChAT SNP and ChEIs treatment response. In addition, Harold et $a l .{ }^{31)}$ failed to find an association with several ChAT SNPs including a SNP $(1882 \mathrm{G}>\mathrm{A})$ which showed complete linkage disequilibrium with a SNP $(2384 \mathrm{G}>\mathrm{A})$, but found an association in only one SNP in promoter region. To the best knowledge of the authors, there has been no previous report that showed an association between a SNP (2384G $>$ A) or exon region and ChEIs response until now. The clinical characteristics of our patients might account for the different results obtained in this study. ChEIs inhibit the acetylcholinesterase from breaking down Ach, thereby increasing both the level and duration of action of the neurotransmitter. Most of the patients in this study were in the early stages of $\mathrm{AD}$, with their clinical severity ranging from very mild to mild by standards of the CDR. The mean score of MMSE-KC increased even after the 26 weeks of donepezil administration in the case of ChAT A carriers. Both the learning effect of cognitive tests or treatment response by ChEIs may have influenced the increase in MMSE-KC scores. This could be especially possible in the high proportion of very mild or mild patients of this study because the clinical effects of ChEIs are generally most conspicuous in the early stages of $\mathrm{AD}$.

Racial differences might be one possible explanation for the discrepancy. ChAT gene polymorphism was associated with AD risk in a dose- or APOE $\varepsilon$ 4-dependent manner among Koreans ${ }^{16-18)}$ and was associated with $\mathrm{AD}$ and mild cognitive impairment in the Chinese. ${ }^{30)}$ However, similar association studies in Caucasians did not find such significant associations. ${ }^{31,32)}$ The role of SNPs in the ChAT gene in relation to risk of AD or response to AD treatment could be different between Asians and Caucasians.

The main advantage of this study was that subjects were recruited in fifteen university hospitals located across Korea. This recruitment on a national scale provided us with both a relatively large sample size and generalizability of our study's results. In addition, we measured two composite cognitive function test scores as well as MMSE scores. We could observe that the differential effect of donepezil by the ChAT genotype was more prominent in verbal memory function measured by CERAD-K(N) WSS than in overall cognitive function measured by CERAD-K(N) TS.

Nevertheless, our results require cautious interpretation because the mechanism by which the ChAT gene influences the treatment effects of ChEIs is still uncertain, and ChAT is not the rate-limiting enzyme for production of Ach. It should also be noted that adequate synthesis of Ach could be maintained until $90 \%$ loss of ChAT activity. ${ }^{33)}$ Although we are far from understanding the exact mechanisms for this differential clinical response, we observed that the ChAT +4 A allele carrying status might influence the treatment effect of donepezil in Koreans.

The number of subjects in this study, however, was not sufficient to have enough power estimates based on power analysis, further researches would be necessary to explore differential clinical responses by other genetic variations of ChAT, which recruit larger sample sizes and diverse ethnic populations.

\section{- Acknowledgments}

This work was supported by the research grant from Eisai Korea and a grant from the Korean Health Technology R\&D Project, Ministry for Health, Welfare, \& Family Affairs, Republic of Korea (Grant No. A092077) .

\section{REFERENCES}

1. Birks J. Cholinesterase inhibitors for Alzheimer's disease. Cochrane Database Syst Rev 2006;(1):CD005593.

2. Hansen RA, Gartlehner G, Lohr KN, Kaufer DI. Functional outcomes of drug treatment in Alzheimer's disease: A systematic review and meta-analysis. Drugs Aging 2007;24: 155-167.

3. Jann MW, Shirley KL, Small GW. Clinical pharmacokinetics and pharmacodynamics of cholinesterase inhibitors. Clin Pharmacokinet 2002;41:719-739.

4. Cacabelos R. Pharmacogenomic protocols in CNS disorders 
and dementia. Neurodegener Dis 2010;7:167-169.

5. Cacabelos R, Martínez R, Fernández-Novoa L, Carril JC, Lombardi V, Carrera I, et al. Genomics of Dementia: $A P O E-$ and CYP2D6-Related Pharmacogenetics. Int J Alzheimers Dis 2012;2012:518901.

6. Pilotto A, Franceschi M, D'Onofrio G, Bizzarro A, Mangialasche F, Cascavilla L, et al. Effect of a CYP2D6 polymorphism on the efficacy of donepezil in patients with Alzheimer disease. Neurology 2009;73:761-767.

7. Seripa D, Bizzarro A, Pilotto A, D'onofrio G, Vecchione G, Gallo AP, et al. Role of cytochrome P4502D6 functional polymorphisms in the efficacy of donepezil in patients with Alzheimer's disease. Pharmacogenet Genomics 2011;21: 225-230.

8. Varsaldi F, Miglio G, Scordo MG, Dahl ML, Villa LM, Biolcati A, et al. Impact of the CYP2D6 polymorphism on steady-state plasma concentrations and clinical outcome of donepezil in Alzheimer's disease patients. Eur J Clin Pharmacol 2006;62:721-726.

9. Bizzarro A, Marra C, Acciarri A, Valenza A, Tiziano FD, Brahe $\mathrm{C}$, et al. Apolipoprotein E epsilon4 allele differentiates the clinical response to donepezil in Alzheimer's disease. Dement Geriatr Cogn Disord 2005;20:254-261.

10. Choi SH, Kim SY, Na HR, Kim BK, Yang DW, Kwon JC, et al. Effect of ApoE genotype on response to donepezil in patients with Alzheimer's disease. Dement Geriatr Cogn Disord 2008;25:445-450.

11. Rigaud AS, Traykov L, Latour F, Couderc R, Moulin F, Forette F. Presence or absence of at least one epsilon 4 allele and gender are not predictive for the response to donepezil treatment in Alzheimer's disease. Pharmacogenetics 2002;12: 415-420.

12. Harold D, Macgregor S, Patterson CE, Hollingworth $\mathrm{P}$, Moore $\mathrm{P}$, Owen MJ, et al. A single nucleotide polymorphism in CHAT influences response to acetylcholinesterase inhibitors in Alzheimer's disease. Pharmacogenet Genomics 2006; 16:75-77.

13. Davies P. Neurotransmitter-related enzymes in senile dementia of the Alzheimer type. Brain Res 1979;171: 319-327.

14. Wilcock GK, Esiri MM, Bowen DM, Smith CC. Alzheimer's disease. Correlation of cortical choline acetyltransferase activity with the severity of dementia and histological abnormalities. J Neurol Sci 1982;57:407-417.

15. Kozak M. Recognition of $A U G$ and alternative initiator codons is augmented by $G$ in position +4 but is not generally affected by the nucleotides in positions +5 and +6 . EMBO J 1997; 16:2482-2492.

16. Lee JJ, Jo SA, Park JH, Lee SB, Jo I, Kim do K, et al. Choline acetyltransferase $2384 G>$ a polymorphism and the risk of Alzheimer disease. Alzheimer Dis Assoc Disord 2012;26:81-87.

17. Ahn Jo S, Ahn K, Kim JH, Kang BH, Kim E, Jo I, et al. ApoE-epsilon 4-dependent association of the choline acetyltransferase gene polymorphisms $(2384 G>A$ and $1882 G>A)$ with Alzheimer's disease. Clin Chim Acta 2006;368:179-182.

18. Kim KW, Suh YJ, Park WY, Jhoo JH, Lee DY, Youn JC, et al. Choline acetyltransferase $G+4$ A polymorphism confers a risk for Alzheimer's disease in concert with
Apolipoprotein E epsilon4. Neurosci Lett 2004;366:182-186.

19. Scacchi R, Gambina G, Moretto G, Corbo RM. Variability of AChE, BChE, and ChAT genes in the late-onset form of Alzheimer's disease and relationships with response to treatment with Donepezil and Rivastigmine. Am J Med Genet B Neuropsychiatr Genet 2009;150B:502-507.

20. McKhann G, Drachman D, Folstein M, Katzman R, Price D, Stadlan EM. Clinical diagnosis of Alzheimer's disease: report of the NINCDS-ADRDA Work Group under the auspices of Department of Health and Human Services Task Force on Alzheimer's Disease. Neurology 1984;34:939-944.

21. Morris JC. The Clinical Dementia Rating (CDR): current version and scoring rules. Neurology 1993;43:2412-2414.

22. Rosen WG, Terry RD, Fuld PA, Katzman R, Peck A. Pathological verification of ischemic score in differentiation of dementias. Ann Neurol 1980;7:486-488.

23. Lee DY, Lee KU, Lee JH, Kim KW, Jhoo JH, Kim SY, et al. A normative study of the CERAD neuropsychological assessment battery in the Korean elderly. J Int Neuropsychol Soc 2004; 10:72-81.

24. Bae JN, Cho MJ. Development of the Korean version of the Geriatric Depression Scale and its short form among elderly psychiatric patients. J Psychosom Res 2004;57:297-305.

25. Sotaniemi M, Pulliainen V, Hokkanen L, Pirttilä T, Hallikainen I, Soininen H, et al. CERAD-neuropsychological battery in screening mild Alzheimer's disease. Acta Neurol Scand 2012;125:16-23.

26. Chandler MJ, Lacritz LH, Hynan LS, Barnard HD, Allen G, Deschner $\mathrm{M}$, et al. A total score for the CERAD neuropsychological battery. Neurology 2005;65:102-106.

27. Park HD, Park KU, Kim KW, Song J, Chang HE, Heo SR, et al. Real-time multiplex PCR assay for genotyping of three apolipoprotein $E$ alleles and two choline acetyltransferase alleles with three hybridization probes. Clin Chem Lab Med 2007:45:346-350.

28. Kim KW, Jhoo JH, Lee KU, Lee DY, Lee JH, Youn JY, et al. Association between apolipoprotein E polymorphism and Alzheimer's disease in Koreans. Neurosci Lett 1999;277:145-148.

29. DeKosky ST, Harbaugh RE, Schmitt FA, Bakay RA, Chui HC, Knopman DS, et al. Cortical biopsy in Alzheimer's disease: diagnostic accuracy and neurochemical, neuropathological, and cognitive correlations. Intraventricular Bethanecol Study Group. Ann Neurol 1992;32:625-632.

30. Tang M, Rao D, Ma C, Guo Y, Han H, Ling $\mathrm{K}$, et al. Evaluation of choline acetyltransferase gene polymorphism (2384 G/A) in Alzheimer's disease and mild cognitive impairment. Dement Geriatr Cogn Disord 2008;26:9-14.

31. Harold D, Peirce T, Moskvina V, Myers A, Jones S, Hollingworth $\mathrm{P}$, et al. Sequence variation in the CHAT locus shows no association with late-onset Alzheimer's disease. Hum Genet 2003;113:258-267.

32. Schwarz S, Eisele T, Diehl J, Müller U, Förstl H, Kurz A, et al. Lack of association between a single nucleotide polymorphism within the choline acetyltransferase gene and patients with Alzheimer's disease. Neurosci Lett 2003;343: 167-170.

33. Haubrich DR, Chippendale TJ. Regulation of acetylcholine synthesis in nervous tissue. Life Sci 1977;20:1465-1478. 